\title{
Solar Wings' 3D Deformation Measurement of Videometrics System
}

\author{
Fengzhe Zhang 1, 2, a, Jiexin Zhou ${ }^{1,2, b}$, Guangcheng Dai ${ }^{3, c}$, Yijie Bian ${ }^{3, d}$, \\ Xia Yang 1, 2, e \\ ${ }^{1}$ College of Aerospace science and Engineering, National University of Defense Technology, \\ Changsha, Hunan, 410073, China \\ ${ }^{2}$ Hunan Provincial Key Laboratory of Image Measurement and Vision Navigation, Changsha, Hunan, \\ 410073, China \\ ${ }^{3}$ College of Computer, National University of Defense Technology, Changsha, Hunan, 410073, \\ China \\ azhangfengzhe163@163.com, bzhoujiexin1993@163.com, cyearto@163.com, \\ dcharlesbyj@vip.qq.com, emr_honson@sina.com
}

\begin{abstract}
Keywords: Solar wings, Videometrics, Binocular stereovision, Camera Calibration, Least squares scene matching, Epipolar constraint.
\end{abstract}

\begin{abstract}
In this paper, we proposed a novel videometrics system application based on binocular stereovision. And we successfully measured three-dimensional (3D) deformation of the solar wings plane. Videometrics has its inherent advantages in solving these problems, which can provide a non-contact, automatic, dynamic and high precision measurement. This system mainly includes camera calibration module, image feature point matching module and 3D model display module. Camera calibration is a key technology of stereoscopic calibration. We applied Zhang Zhengyou new approach, which is widely used to calibrate the camera. For the image matching part, we used the correlation matching method and Ackerman Least Squares method, which can achieve accuracy of $1 / 10$ or even 1/100 pixel. To further optimize the process, epipolar constraint was adopted. Two-dimensional search can be converted into a one-dimensional search, thereby highly reducing the matching computation. Moreover, 3D space measured results obtained in the target point test can be displayed in the system. With the target region segmentation statistics, the correct result is more vividly displayed and accurately measured. Based on the proposed method we have designed the measurement software and simulate the solar wings's 3D deformation measurement experiment. The re-projection error for both of stereo cameras is no more than 0.2 pixel. The height difference of solar wings plane is $0.014 \mathrm{~mm}$. The method proposed in this paper has significance both on the theory and application.
\end{abstract}

\section{Introduction}

Solar wings is the main energy source for satellite. With the rapid development of aerospace industry, the task undertaken by the satellites is becoming increasingly heavier. The 3D deformation measurement of solar wings is of great significance for structural strength analysis, vibration suppression and motion control. And the demand for energy is also increasing. Limited by the launch condition as well as the producing cost, the satellite solar wing inevitably tends to be large but light, which makes it a large-span, light-weighted and low-rigidity spatial structure. In outer space where there is no resistance force for satellites, any external or internal interference can cause intense sustained substantial vibration of solar wings. So vibration and structural strength of the solar wing issues must be taken seriously [1-3].

This paper mainly studied the solar wings' three dimensional deformation measurement videometrics system, based on the application problem of videometrics. We also provided basis to solve problems of solar wings' vibration and structural strength in order to offer timely warning for damaged deformed solar wing. 


\section{Principle}

\subsection{The basic principle of binocular stereo vision}

The binocular stereo vision is observing the same object from two viewpoints. According to the projection model, pixel position deviation of the two picture can be calculated, and the three dimensional information of the object can be obtained.

Fig. 1 shows the basic principle of binocular stereovision, in which, left camera optical center $O_{l}$ is , the origin of the coordinate system in binocular stereo vision system; since the direction of $\mathrm{X}$-axis and $\mathrm{Y}$-axis is shown in Fig.1, the Z-axis direction can be determined by right-hand principle. Two cameras are observing a feature point $p\left(x_{c}, y_{c}, z_{c}\right)$ of the object at the same moment, and coordinate points $p_{1}\left(x_{1}, y_{1}\right)$ and $p_{2}\left(x_{2}, y_{2}\right)$ are obtained in the left and the right image.

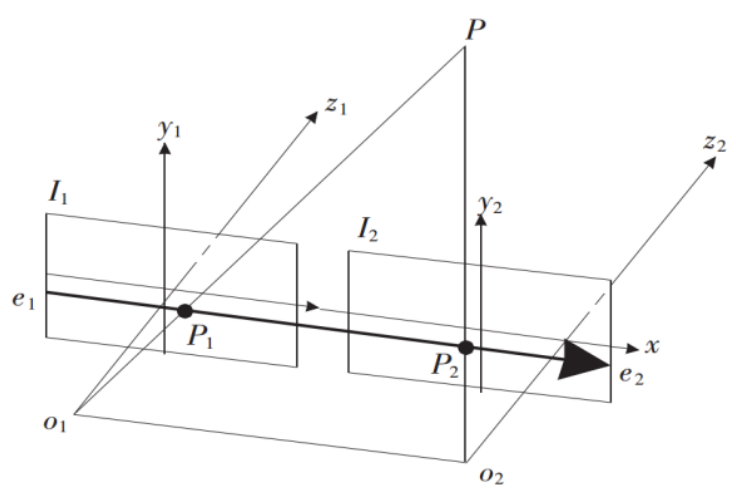

Fig. 1 Epipolar geometry

Assuming the images from the two cameras are on the same plane, then the points projected by the feature point in the two images have the same Y-axis coordinates. According to the triangular geometrical relations, we obtain:

$$
x_{l}=f \cdot x_{c} / z_{c}, x_{r}=f \cdot\left(T-x_{c}\right) / z_{c}, y=f \cdot y_{c} / \mathbf{z}_{c}
$$

Setting the optical parallax $d=x_{l}-x_{r}$, then according to the similar triangle theorems, coordinates of the feature point in the binocular stereo vision system can be calculated:

$$
x_{c}=d_{o_{1} o_{2}} \cdot x_{l} / d, y_{c}=d_{o_{1} O_{2}} \cdot y / d, z_{c}=d_{o_{1} O_{2}} \cdot f / d
$$

In the following parts, this paper first introduces the epipolar geometric constraints on the images, through which, for any point in the left camera image plane, as long as we can find the corresponding match point in the right camera image plane, the 3D coordinates of this point can be determined.

\subsection{Epipolar constraint principle}

Using multi-view geometric constraints, we considered the relative geometric relationship between the images to be matched on the basis of the imaging characteristics of the target. Through feature point matching according to the disparity epipolar constraint condition, two-dimensional search can be converted into a one-dimensional search, thereby reducing the match computation. Using geometric constraints from the object side and the image side, such as the extrinsic parameters of the camera parameters and the geometry of the object, the searching range can be efficiently narrowed. Making full use of these constraints, we can not only improve the efficiency of matching search, but also enhanced matching reliability under the circumstances of occlusion, surface discontinuities, etc.

In the imaging system of the binocular stereo vision, the connecting line between the two optical centers of the two cameras is called the baseline. And all the planes which include the two optical centers are called the polar plane. To give a point in space outside the baseline, the given point together with the baseline can determine a specific polar plane. The intersection line between the stereographic camera image plane and the polar plane is called the epipolar line. The intersection point of the baseline and the image plane is called a pole. As Fig. 2 shows, a spatial point $\mathbf{X}$, whose homogeneous coordinates is $\mathbf{P}$, is projected to in the image planes $\mathbf{S}$ and $\mathbf{S}^{\prime}$ of the two cameras, the optical center of which are $\mathbf{C}$ and $\mathbf{C}^{\prime} . \mathbf{P 1}$ and $\mathbf{P 2}$ are the homogeneous coordinates of the projection points of $\mathbf{P}$ in $\mathbf{S}$ and $\mathbf{S}^{\prime}$. The space plane $\pi$ determined by $\mathbf{C}, \mathbf{C}^{\prime}$ and $\mathbf{X}$ is a polar plane including $\mathbf{X}$. $\mathbf{I}$ 
and l', intersected by the image plane $\mathbf{S}$ and $\mathbf{S}^{\prime}$ with plane $\pi$, are epipolar lines. $\mathbf{e}$ and $\mathbf{e}^{\prime}$, intersected by $\mathbf{S}$ and $\mathbf{S}^{\prime}$ with the baseline are poles. The points, lines, and planes mentioned above are all expressed in their vector form. It is not hard to find that, corresponding points $\mathbf{P 1}$ and $\mathbf{P} 2$ of the spatial point $\mathbf{X}$ in the image plane must lie in the corresponding polar lines $\mathbf{I}$ and $\mathbf{l}^{\prime}$. The very geometry of such a relationship between two images, is the epipolar constraint.

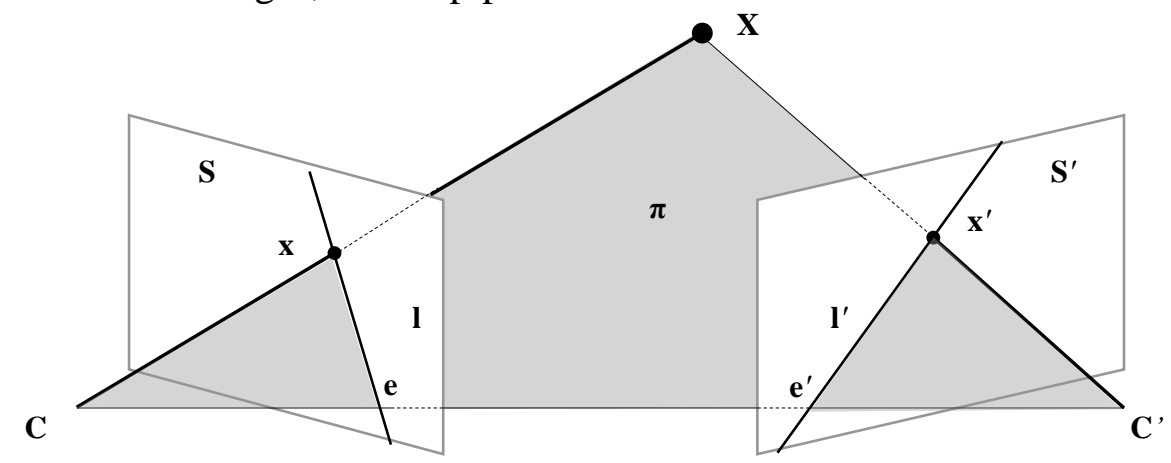

Fig.2 Epipolar constraint relation

The epipolar constraint relation between two images can be expressed by fundamental matrix. The deduction of the fundamental matrix is introduced as follows.

Given $\mathrm{K}$ and $\mathrm{K}^{\prime}$ are the camera intrinsic parameter matrixes of two imaging processes:

$$
K=\left[\begin{array}{ccc}
F_{x} & 0 & C_{x} \\
0 & F_{y} & C_{y} \\
0 & 0 & 1
\end{array}\right], K^{\prime}=\left[\begin{array}{ccc}
F_{x}^{\prime} & 0 & C_{x}^{\prime} \\
0 & F_{y}^{\prime} & C_{y}^{\prime} \\
0 & 0 & 1
\end{array}\right]
$$

In (3), $\left(C_{x}, C_{y}\right),\left(C_{x}^{\prime}, C_{y}^{\prime}\right)$ and $\left(F_{x}, F_{y}\right),\left(F_{x}^{\prime}, F_{y}^{\prime}\right)$ are the optical centers and equivalent focal lengths of two imaging processes. Given the projection matrixes of the imaging are $\mathbf{M}$ and $\mathbf{M}^{\prime}$, according to the imaging relation, we get

$$
\lambda x=M x, \lambda^{\prime} x^{\prime}=M^{\prime} x
$$

$\lambda, \lambda^{\prime}$ stand for the distance between the spatial point and the optical center $\mathbf{C}$ and $\mathbf{C}^{\prime}$. Given $\mathbf{M}^{+}$is the pseudo-inverse of $\mathbf{M}, \mathbf{M} * \mathbf{M}^{+}$is, therefore, a unit matrix. Then the spatial point $\mathbf{X}$ can be expressed as

$$
\mathbf{X}=\lambda M^{+} \mathbf{x}
$$

Where $\lambda$ is the distance between $\mathbf{X}$ and the optical center $\mathbf{C}$. substitute (5) into the second equation in (4), we get $\mathbf{x}$ 's corresponding image point $\mathbf{x}^{\prime}$,

$$
\mathbf{x}^{\prime}=\frac{\lambda}{\lambda^{\prime}} M^{\prime} M^{+} \mathbf{x}
$$

Where $\lambda^{\prime}$ is the distance between $\mathbf{X}$ and optical center $\mathbf{C}^{\prime}$. The pole $\mathbf{e}^{\prime}$ is the corresponding image point of the first imaging optical center $\mathbf{C}$ in the second

$$
\mathbf{e}^{\prime}=\frac{1}{\lambda_{e}} M^{\prime} \mathbf{C}
$$

Where $\lambda_{e}$ is the distance between the two optical center $\mathbf{C}$ and $\mathbf{C}^{\prime}$. Epipolar line $\mathbf{l}^{\prime}$ passes through $\mathbf{e}^{\prime}$ and $\mathbf{x}^{\prime}$, so

$$
\mathbf{l}^{\prime}=\left[\mathbf{e}^{\prime}\right]_{\times} \mathbf{x}^{\prime}=\frac{\lambda}{\lambda^{\prime}}\left[\mathbf{e}^{\prime}\right]_{\times} M^{\prime} M^{+} \mathbf{x}
$$

Where $\left[\mathbf{e}^{\prime}\right]_{\times}$is the anti-symmetric matrix of the rank of $\mathbf{e}^{\prime}=\left[\begin{array}{lll}e_{0} & e_{1} & e_{2}\end{array}\right]^{T}$, also known as Cross Product. 


$$
[\mathbf{e}]_{\times}=\left[\begin{array}{ccc}
0 & -e_{2} & e_{1} \\
e_{2} & 0 & -e_{0} \\
-e_{1} & e_{0} & 0
\end{array}\right]
$$

Since the image point $\mathbf{x}^{\prime}$ is on the epipolar line $\mathbf{l}^{\prime}$,

$$
\mathbf{x}^{\prime \mathbf{T}} \mathbf{l}^{\prime}=0
$$

Substitute (8) to (10),we get

$$
\mathbf{x}^{\prime \mathbf{T}} \mathbf{l}^{\prime}=\frac{\lambda}{\lambda^{\prime}} \mathbf{x}^{\prime \mathbf{T}}\left[\mathbf{e}^{\prime}\right]_{\times} M^{\prime} M^{+} \mathbf{x}=0
$$

Name $\mathbf{F}$ a fundamental matrix,

$$
\mathbf{F} \equiv\left[\mathbf{e}^{\prime}\right]_{\times} M^{\prime} M^{+}
$$

Then we describe the epipolar constraint relation between two images using the fundamental matrix $\mathbf{F}$,

$$
\mathbf{x}^{\prime \mathbf{T}} \mathbf{F} \mathbf{x}=0
$$

The rank of $\left[\mathbf{e}^{\prime}\right]_{\times}$is 2 and the rank of $M^{\prime} M^{+}$is 3 . Therefore, the fundamental matrix $\mathrm{F}$ is a $3 \times 3$ matrix with the rank of 2 .

\subsection{Epipolar correction algorithm}

The ultimate goal of the camera calibration and correction is to make the two camera optical axes parallel for the further depth calculation and 3D measurement. In this paper, we use epipolar correction algorithm of Bouguet's in opencv.

The optical axes of left camera and the right one are not perfectly parallel before correction. The intersection point of the image plane and the baseline is the pole. Lines that passes through the image points and the pole are the polar lines. And the polar plane is the plane that includes the left and the right epipolar line as well as the baseline.

After correction, the pole is at infinity. And the optical axes of left camera and the right one get parallel, this is, the image point is at the same height in both left and right images, which is the goal of epipolar correction. For the following stereo matching, we just need to search in the one line, lowering the searching dimension from $O\left(\mathrm{n}^{2}\right)$ to $O(\mathrm{n})$.

Bouguet method is generally, concerting the Rotation matrix and the Translation matrix calculated by OpenCV into half-rotation situation for both the left and the right camera. In this method, the Rotation matrix and the Translation matrix are resolved into $R_{1}, T_{1}, R_{2}, T_{2}$, during which, the principle is to keep the least distortion in the re-projection of left and right images, and to have the biggest common area of the left and the right image.

\section{Videometric System Calibration}

The method used in this paper is a new, flexible approach between traditional method and self-calibration method called Zhang's plane calibration method proposed by Zhang Zhengyou new approach $^{[2]}$. Since the method is already quite mature and popular, the basic steps of Zhang Zhengyou camera calibration method are given below.

1. Print the chessboard pattern, then paste it on a plane. The chessboard used in this paper is $16 \times 12$ grid, which is made up with $3 \times 3 \mathrm{~cm}$ squares.

2. Take pictures of the pattern from different angles by moving the camera or the pattern. In this paper, we took 16 calibration pictures using binocular cameras from different angles.

3. Detect the corner information of the images.

4. Calculate the homography matrix $\mathrm{H}$ of every image.

5. Working out the intrinsic and extrinsic parameter matrixes using $\mathrm{H}$, giving the distortion coefficient is 0 . Then calculate the distortion coefficient using linear least square method ${ }^{[3]}$.

6. Optimize all the parameters using minimization method. 


\section{Image feature point matching}

\subsection{Correlation matching principle}

Correlation algorithm is to select the central pixel of a pixel window with the maximum correlation coefficient and greater than the set threshold as the very point to be extracted, through individually calculating the similarity of the template image and the measured image of a certain size of pixel windows, with the correlation coefficient as a matching measure. The correlation coefficient is a normalized covariance function. Supposing the coordinates of the target center of the template image pixel is $(i, j)$, the measured image search center pixel coordinates is $(i+\alpha, j+\beta)$, this formula for two specific window correlation coefficient is calculated as follows:

$$
\rho(\alpha, \beta)=\frac{\sum_{i=1}^{m} \sum_{j=1}^{n}\left(g_{i j}-\bar{g}\right)\left(g_{i+\alpha, j+\beta}-\bar{g}_{i+\alpha, j+\beta}^{\prime}\right)}{\left[\sum_{i=1}^{m} \sum_{j=1}^{n}\left(g_{i j}-\bar{g}\right)^{2}\left(g_{i+\alpha, j+\beta}-\bar{g}_{i+\alpha, j+\beta}^{\prime}\right)^{2}\right]^{1 / 2}}
$$

Where $\bar{g}$ and $\bar{g}^{\prime}$ are all arithmetic mean values.

For a one-dimensional correlation, $\beta=0$. If the maximum value of the correlation coefficient greater than a threshold in one measured image search window, it is considered the center pixel of the search window is the match point. In the viewpoint of geometry, the value of the correlation coefficient between the two vectors is equal to the cosine of the angle. The smaller the angle between two vectors the greater the similarity between the two windows, so the linear correlation coefficient can be described as the degree of similarity between the two windows.

\subsection{Conditions for extreme existence of Least Squares gray-scale difference measurement function}

In the image matching, it may occur that the system is not be able to obtain a correct match. For example, for three points on the left in Fig. 3, only point 1 has the one and only corresponding matching point in the right figure. Therefore, in order to ensure the reliability of the matching algorithm, we need to study the necessary and sufficient conditions when the extreme value of match measure functions exists.

Supposing $f(\mathrm{x}, \mathrm{y})$ and $\mathrm{g}(\mathrm{x}, \mathrm{y})$ are the equal-sized local feature images of to-be-matched images $I_{1}(x, y)$ and $I_{2}(x, y)$, we normalized the mean gray value of local feature images, making the sum of gray scale of the local images zero. Regard the minimum quadratic sum of gray difference as match measure, and the matching problem is converted into the following optimization problem.

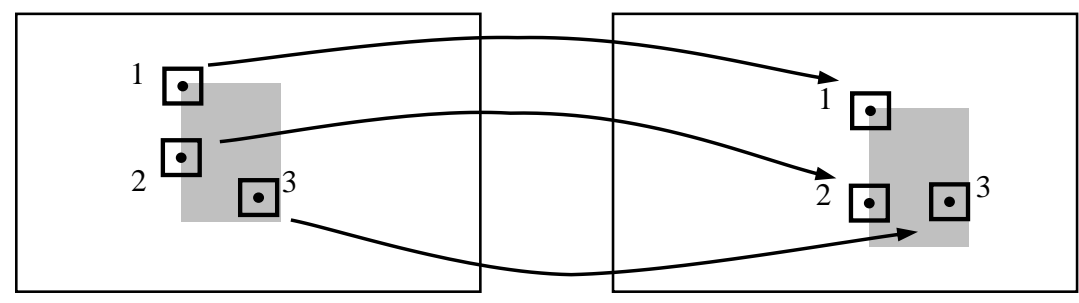

Fig.3 Image matching exists

$$
\min _{(u, v)} M(u, v)=\min _{(u, v)} \sum_{(x, y) \in W}[f(x, y)-g(x+u, y+v)]^{2}
$$

Where $\mathrm{W}$ is the local area of feature $f(\mathrm{x}, \mathrm{y}),(u, v)$ is for the coordinates transformation of the matching feature. To simplify the deduction, the summation is denoted as $\Sigma$.

Expanding the match meaturement function $M(u, v)$ and combining the variables, we get

$$
M(u, v)=\left(\sqrt{\sum f^{2}}-\sqrt{\sum \hat{g}^{2}}\right)^{2}+2 \sqrt{\sum f^{2} \sum \hat{g}^{2}}\left(1-\frac{\sum f \hat{g}}{\sqrt{\sum f^{2} \sum \hat{g}^{2}}}\right)
$$


In (16), $f$ and $\hat{g}$ stands for function $f(\mathrm{x}, \mathrm{y})$ and $g(x+u, y+v)$. The correlation coefficient $\rho$ of image $f(\mathrm{x}, \mathrm{y})$ and $g(x+u, y+v)$ is given below.

$$
\rho=\frac{\sum f * \hat{g}}{\sqrt{\sum f^{2} \sum \hat{g}^{2}}}
$$

Then (16) can be transformed into,

$$
M(u, v)=\left(\sqrt{\sum f^{2}}-\sqrt{\sum \hat{g}^{2}}\right)^{2}+2(1-\rho) \sqrt{\sum f^{2} \sum \hat{g}^{2}}
$$

As can be known from (18), when the correlation coefficient reaches the maximum, $M(u, v)$ gets the minimum value. So, match measure of the minimum quadratic sum of gray difference and of the maximum correlation coefficient agrees with each other. The necessary and sufficient conditions when the extreme value of match measure functions exists, are discussed below, in an ideal situation.

Let's assume there is a first-order continuous partial derivatives for $u$ in the neighborhood of point $(x, y)$, Expand $g(x+u, y+v)$ at the point $(x, y)$, and retain first-order item, we get

$$
\begin{aligned}
& g(x+u, y+v) \approx g(x, y)+g_{x} u+g_{y} v \\
& g_{x}=g_{x}^{\prime}(x, y), \quad g_{y}=g_{y}^{\prime}(x, y)
\end{aligned}
$$

Substitute (20) into (19),

$$
\begin{aligned}
M(u, v) \approx & \left(\sum g_{x}^{2}\right) u^{2}+2\left(\sum g_{x} g_{y}\right) u v+\left(\sum g_{y}^{2}\right) v^{2}- \\
& 2\left(\sum d g_{x}\right) u-2\left(\sum d g_{y}\right) v+\sum d^{2}
\end{aligned}
$$

Where $d$ represents function $d(x, y)=f(x, y)-g(x, y)$.

As can be seen from (21), the match measure $M(u, v)$ can be approximated as a quadratic function, namely a space surface. For different image features, $M(u, v)$ does not necessarily have extreme . According to the nature of the quadratic function, the necessary and sufficient conditions for the extreme for function $M(u, v)$ is,

$$
\left[\sum g_{x}^{2} \sum g_{y}^{2}-\left(\sum g_{x} g_{y}\right)^{2}\right]>0
$$

Calculating the first-order partial derivatives of function $Y(u, v)$ with respect to the variables $u$ and set the derivatives zero. We can obtain the following equation.

$$
\left\{\begin{array}{l}
\left(\sum g_{x}^{2}\right) u+\left(\sum g_{x} g_{y}\right) v=\sum d g_{x} \\
\left(\sum g_{x} g_{y}\right) u+\left(\sum g_{y}^{2}\right) v=\sum d g_{y}
\end{array}\right.
$$

When (22) is ture, (23) has real solution, namely the extreme points. When $\Sigma d g x=\Sigma d g y=0$, $(0,0)$ is an extreme point for function $M(u, v)$. For gray-scale feature, we have

$$
\left(\sum g_{x}^{2}+\sum g_{y}^{2}\right)>0
$$

So, the minimal value of function $M(u, v)$ exists

\subsection{The basic idea of the least squares matching}

Shooting the same scene using two calibrated cameras, we get two images $I_{1}, I_{2}$. We take $I_{1}$ as the reference template image, $I_{2}$ as measured image. Given that template gray scale is $f(x, y)$, the corresponding point is denoted as $\left(x_{f}, y_{f}\right)$. For measured image matching window gray scale $g(x, y)$, the corresponding point is denoted as $\left(x_{g}, y_{g}\right)$. The affine transformation between the template and the matching image is, 


$$
\left\{\begin{array}{l}
x_{g}=a_{0}+a_{1} x_{f}+a_{2} y_{f} \\
y_{g}=b_{0}+b_{1} x_{f}+b_{2} y_{f}
\end{array}\right.
$$

The purpose of matching is to calculate the transformation parameters $\left(a_{0}, a_{1}, a_{2}, b_{0}, b_{1}, b_{2}\right)$, combine the precise coordinates of edge points given on the template, we can get the exact position of the edge points.

Least squares method is an iterative process, the first step of which is to roughly extract results as the initial iteration value of parameter transformation and get deformation parameters . Re-sample the measured image using the corrected parameters, and we calculate the correlation coefficient between the template and the matching image. If the coefficient is greater than a predetermined threshold, the iteration ends. In order to get a better iteration result, in the subsequent programming, we can do some basic image processing operations first to highlight features, during which, the operators, including histogram equalization, median filter, Sobel edge detection etc., are used to enhance texture image. Then we use the linear affine transformation, and the quadric surface affine transformation for a coarse correlation matching template and a fine correlation matching, which allows us to obtain the initial values of the positions of corresponding points after a coarse registration. In the end, we use the iterative least squares adjustment calculation, which can reach $1 / 10$ and 1/100 pixel matching accuracy, confirming corresponding points of sub-pixel.

\section{Experiment and Results}

\subsection{Experiment system structure and composition}

System structure. Based on binocular stereo vision camera intersection measurement principle, the overall design scheme and system structure are shown in Fig. 4.

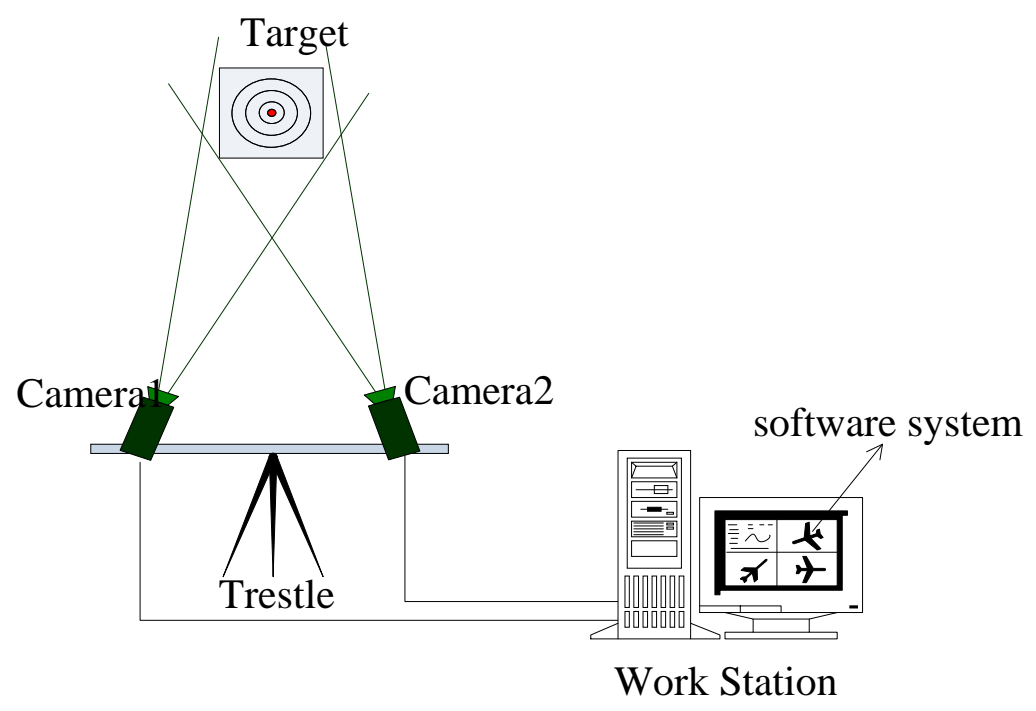

Fig.4 The overall experiment design

According to the actual shooting, the solar wing panel size, is approximately $70 \mathrm{~cm} \times 30 \mathrm{~cm}$. Calibration grid pattern horizontal and vertical corner points is $16 \times 12$, the size of square is $3 \mathrm{~cm} \times 3 \mathrm{~cm}$, as shown in Fig. 5 .

In order to make full use of the field of view, we selected $16 \mathrm{~mm}$ lens for the left and right camera. Correctly connecting the two camera power lines, Gigabit network acquisition card, and calibrating network port IP information, we can initialize the camera in the workstation software system, and display of the real-time image.

Hardware components:

In order to achieve the goal of imaging, image acquisition, image storage and other functions, it is necessary to set up a binocular camera measurement system, the main hardware components are as following. 
Two DMK72A (B)UC02 grayscale cameras, with a resolution of $2592 \times 1944$ pixel and frame rate $6 \mathrm{fps}$ to achieve the target imaging and image acquisition.

A computer, used to control the camera synchronization trigger, and realize of the image storage and calculation, to get the simulated scene images.

Two video lines, one connected to the camera video output port, one connected to the computer USB port. And the other two ends are connected to the image acquisition card which is fixed on the main board of the computer PCI slot.

Image acquisition card, to digitalize the image, and transfer into computer memory for further computer processing.

High precision calibration board. The size is depending on the need to measure the size of the object. And the calibration board used in this experiment is the size of $16 \times 12$ square grid, the size of each small square is $3 \mathrm{~cm} \times 3 \mathrm{~cm}$.

Satellite solar wing model. In order to make the environment more real, the solar wing with the length of $1.5 \mathrm{~m}$ and width of $0.3 \mathrm{~m}$, which is from small satellite model in the laboratory, is used.

Select 16 calibration board images each shoot by the left and the right cameras, and input them into the software.The Calibration Calculation, Matching Calculation, and 3D Display Unit are shown in Figure 6 and Figure 7.
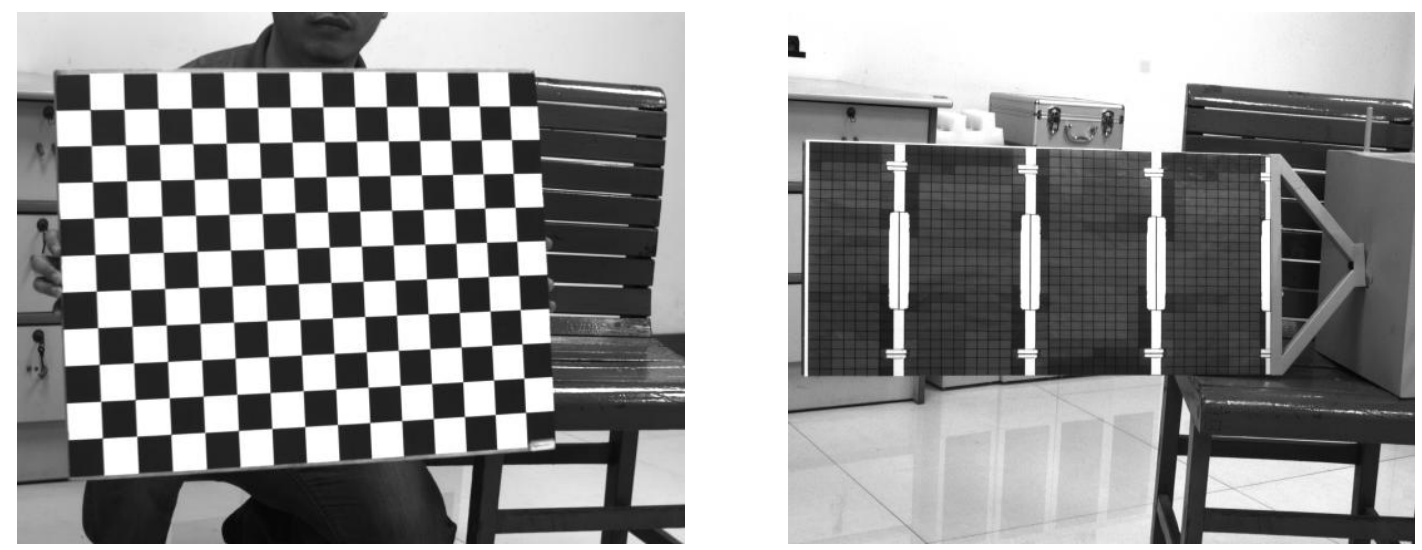

Fig. 5 Left: calibration pattern; Right: Simulated solar wing panels
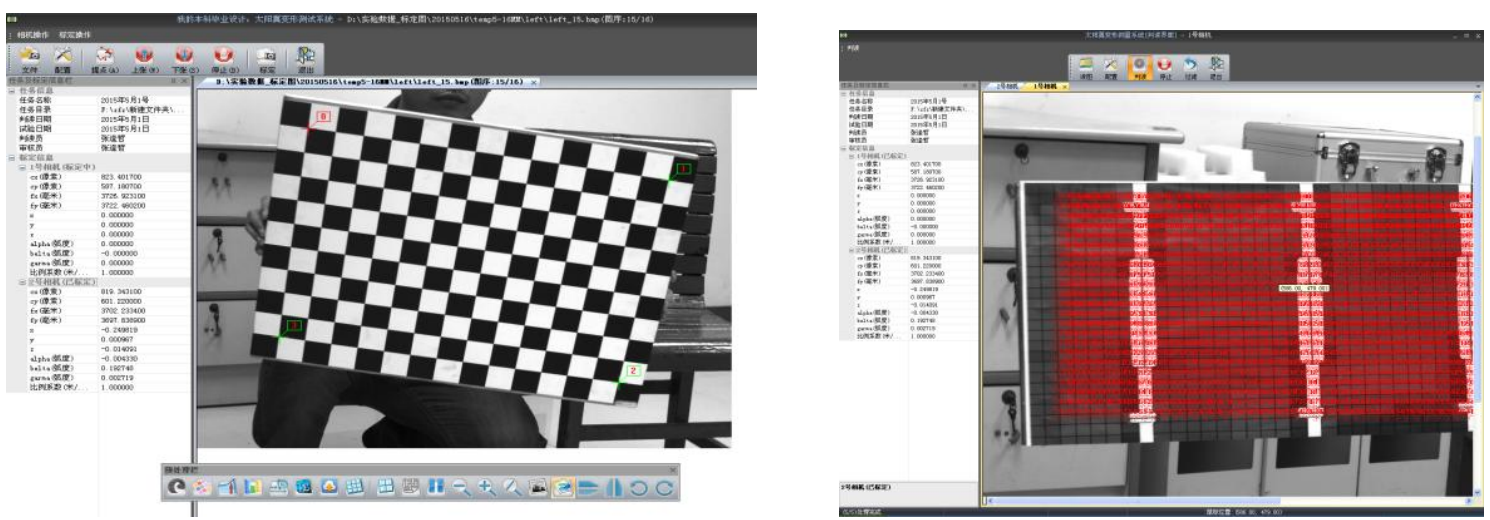

Fig. 6 Left: Calibration calculation unit; Right: Matching calculation unit 


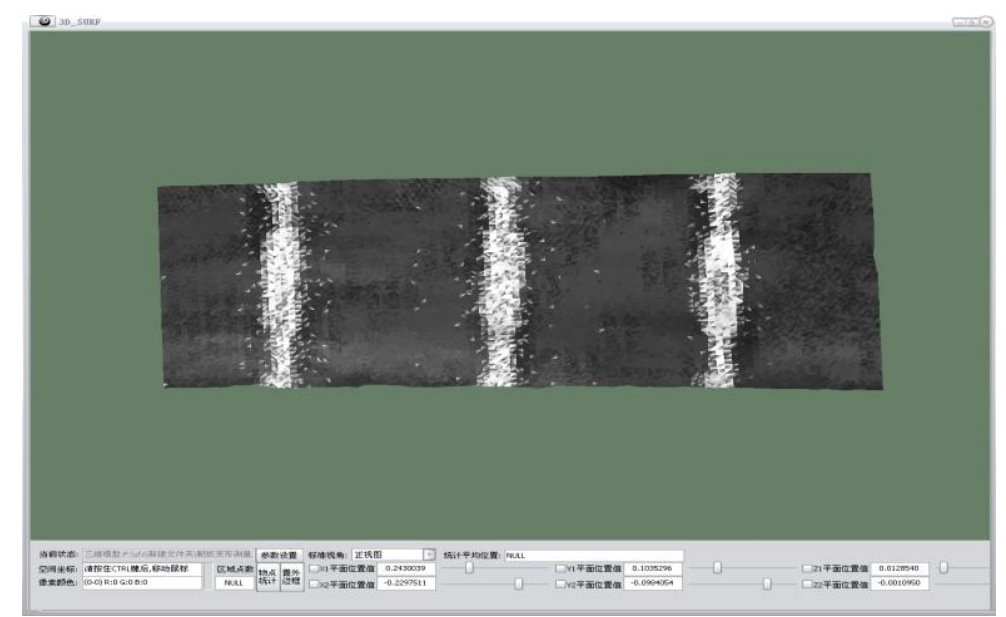

Fig. 7 3D display unit

\subsection{Experimental results and analysis}

Calibration result and analysis. We shot the high precision calibration board with binocular cameras, and chose 16 calibration images from each of the left and right camera which are required in the camera specific imaging range, from different angles. Then input the images into the measurement software.

The calibration information includes the image center $(c x, c y)$, equivalent focal length $\left(f_{x}, f_{y}\right)$, distortion coefficient $(\mathrm{k} 1, \mathrm{k} 2, \mathrm{p} 1, \mathrm{p} 2, \mathrm{~S})$, camera coordinates $(\mathrm{x}, \mathrm{y}, \mathrm{z})$, and camera angle parameters (alpha, belta, garma). The final calibration result is in Table1.

Table 1 Camera intrinsic and extrinsic parameters and distortion coefficient

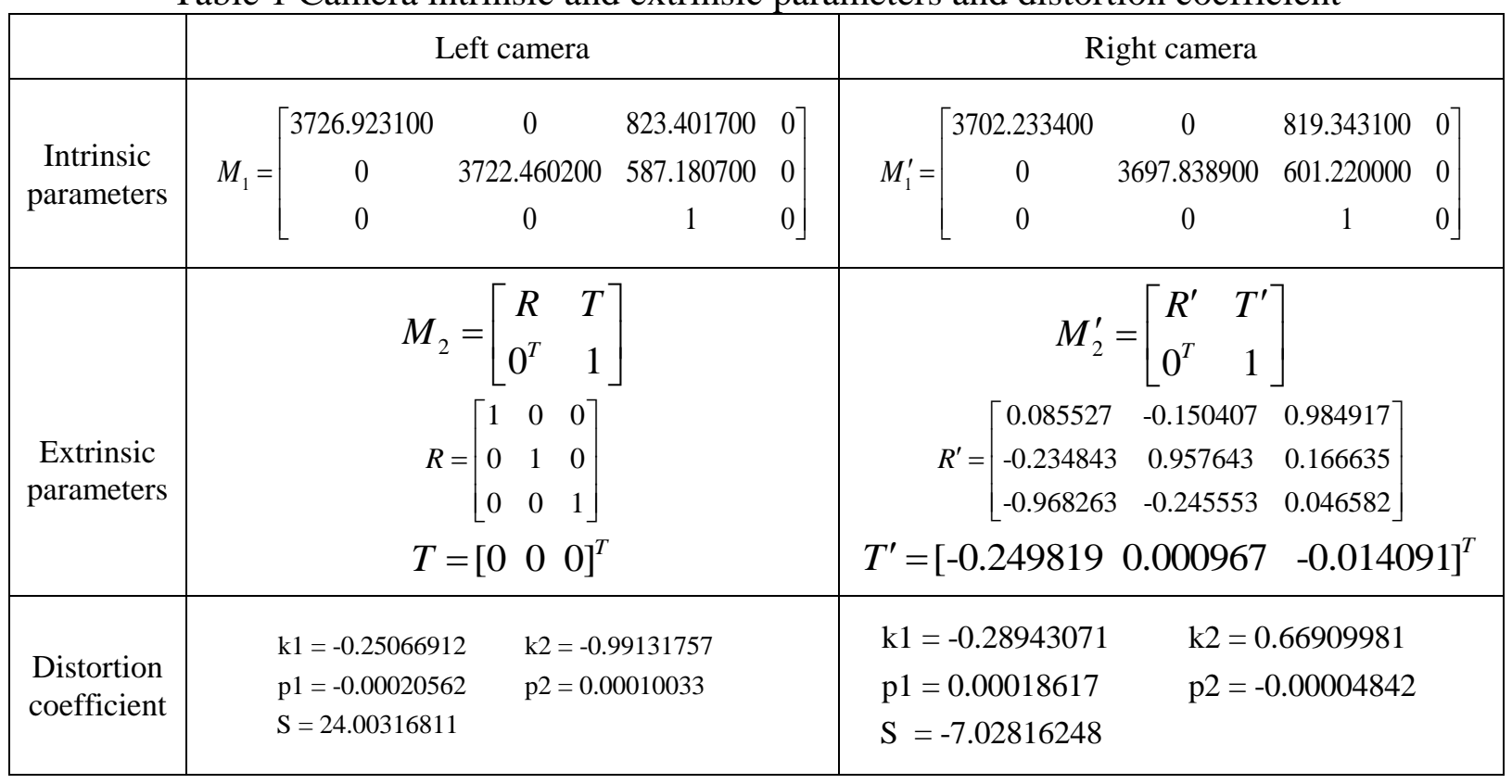

The taskbar and calibration information bar display task information and calibration information of the left and right camera (No. 1 and No. 2 camera). The ".txt" file output from the right shows, 16 images are all calibrated successfully. During the calibration process, the corner points of coarse search and fine search is the $14 \times 10$ grid corner points extracted from the calibration plate with a total number of 140 points. Processing re-projection residual error $\delta$, we can calculate the mean square error between the ideal imaging position $\left(x_{i j}, y_{i j}\right)$ and the distortion-corrected image position $\left(\tilde{x}_{i j}, \tilde{y}_{i j}\right)$, via (26). 


$$
\left\{\begin{array}{l}
\delta_{x}=\sqrt{\sum_{i, j}\left(x_{i j}-\tilde{x}_{i j}\right)^{2} / N-1} \\
\delta_{y}=\sqrt{\sum_{i, j}\left(y_{i j}-\tilde{y}_{i j}\right)^{2} / N-1} \\
\delta=\sqrt{\delta_{x}^{2}+\delta_{y}{ }^{2}}
\end{array}\right.
$$

For example, the residual error of the first image contains two parts, for the left camera 0.13682 pixel ,and the right camera 0.122701 pixel. It is found that every residual error for 16 images from the left and right camera is no more than 0.2 pixel, which shows that the calibration accuracy is within the normal range.

Matching results and analysis. Input the matching image from both cameras, which is the two solar wing panel image shot at the same moment by the binocular cameras (Fig. 8).
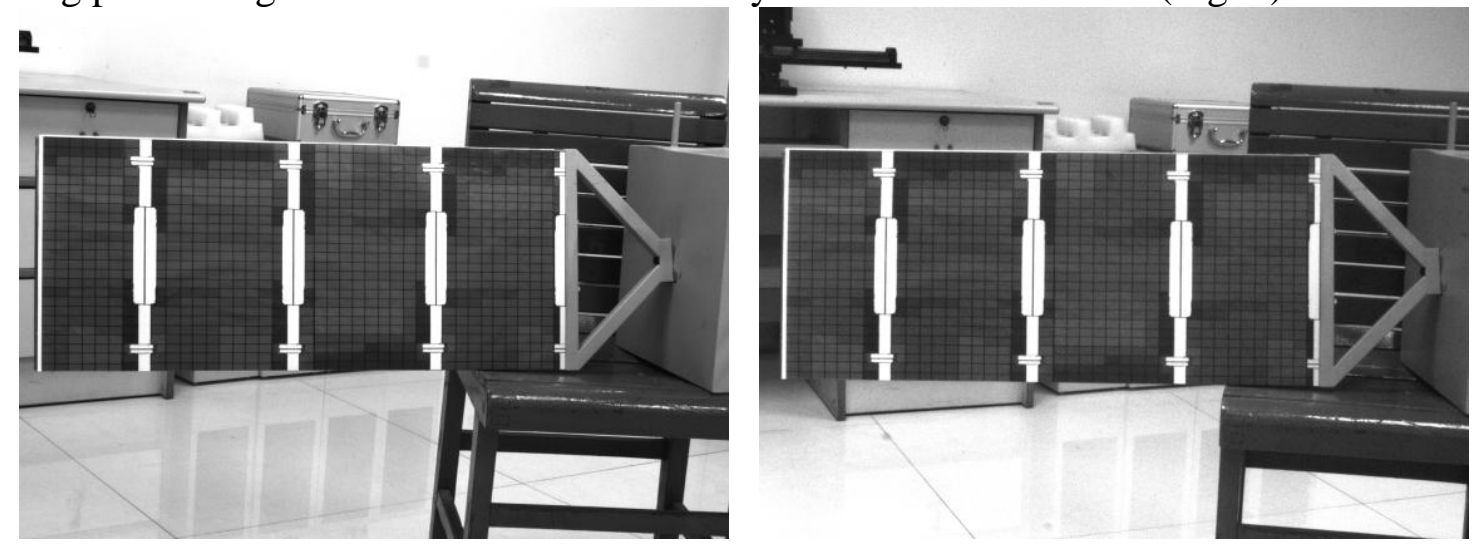

Fig. 8 The solar wing panel images shot by left and right camera

Set the interpretation parameters, including the interpretation mode, whether to display the matching point, the template radius, the search radius and the distance between the points. The reasonable selection of the template radius, search radius and the distance between the points can ensure the speed and accuracy of the matching. Based on the experience, we can set the template radius 60 , the search radius 40 , and the step length 10 .

Select 4 stable points from each image in Fig.9, and start the interpretation. The points' pixel coordinates are listed in Table 2.

Table 2 The selected stable points' pixel coordinates

\begin{tabular}{|c|cc|}
\hline Left camera & $(111.700000,314.900000)$ & $(1186.300000,317.900000)$ \\
& $(124.100000,811.600000)$ & $(1206.200000,773.700000)$ \\
\hline \multirow{2}{*}{ Right camera } & $(234.900000,350.600000)$ & $(1352.200000,344.600000)$ \\
& $(241.600000,841.500000)$ & $(1367.100000,817.000000)$ \\
\hline
\end{tabular}

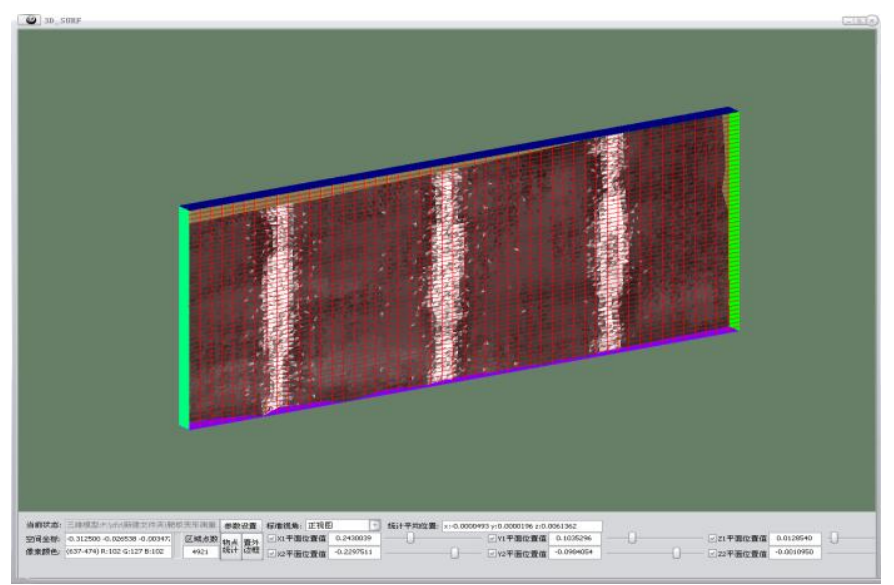

Fig. 9 Framed solar wing panel model 
Enter matching interpretation for the selected points, and "filter" the interpretation result, cutting out the points with big error.

The size of the image is $1600 \times 1200$ pixels, with a total number of 4922 matching points. From the beginning of the second row, each line represents the data of a matching point. For each column, from left to right, information are: $\mathrm{x}$ coordinates of matching points in the left camera image, $\mathrm{y}$ coordinates in the left camera image, $x$ coordinates in the right camera image, $y$ coordinates in the right camera image, $x$ component of spatial coordinates of the matching points, $y$ component of spatial coordinates, and $\mathrm{z}$ component of spatial coordinates of the matching points (the left camera is regarded as the world coordinate origin). The last three columns are the RGB value of the matching points.

3-D display and deformation analysis

Having obtained the matching data of solar wing panels correspondence points, we can build the 3D model using the 3D display module. Frame the surface imaging model with rectangular and display it from three view angles, as shown in Table 3.

Table 3 Matching result

\begin{tabular}{|c|c|c|c|c|c|c|c|c|c|}
\hline $\begin{array}{c}\text { x coordinates } \\
\text { in left camera }\end{array}$ & $\begin{array}{c}\text { y coordinates } \\
\text { in left camera }\end{array}$ & $\begin{array}{c}\text { x coordinates } \\
\text { in right } \\
\text { camera }\end{array}$ & $\begin{array}{c}\text { y coordinates } \\
\text { in right } \\
\text { camera }\end{array}$ & $\begin{array}{c}\text { x coordinates } \\
\text { in space }\end{array}$ & $\begin{array}{c}\text { y coordinates } \\
\text { in space }\end{array}$ & $\begin{array}{c}\text { z coordinates } \\
\text { in space }\end{array}$ & $\mathrm{R}$ & $\mathrm{G}$ & $\mathrm{B}$ \\
\hline 124.0000 & 774.0000 & 241.9885 & 803.7707 & 0.0007 & 0.1888 & 0.0059 & 56 & 56 & 56 \\
\hline 134.0000 & 774.0000 & 252.1195 & 803.7845 & 0.0049 & 0.1883 & 0.0066 & 70 & 70 & 70 \\
\hline 144.0000 & 774.0000 & 262.2452 & 803.7861 & 0.0090 & 0.1884 & 0.0073 & 64 & 64 & 64 \\
\hline 154.0000 & 774.0000 & 272.1292 & 803.7791 & 0.0131 & 0.1885 & 0.0073 & 60 & 60 & 60 \\
\hline 164.0000 & 774.0000 & 281.9212 & 803.7767 & 0.0171 & 0.1886 & 0.0071 & 72 & 72 & 72 \\
\hline 174.0000 & 774.0000 & 291.8324 & 803.7509 & 0.0211 & 0.1886 & 0.0071 & 76 & 76 & 76 \\
\hline 184.0000 & 774.0000 & 301.7988 & 803.7312 & 0.0252 & 0.1887 & 0.0073 & 47 & 47 & 47 \\
\hline 194.0000 & 774.0000 & 311.5367 & 803.7171 & 0.0293 & 0.1888 & 0.0068 & 76 & 76 & 76 \\
\hline 204.0000 & 774.0000 & 321.5747 & 803.7056 & 0.0334 & 0.1890 & 0.0071 & 72 & 72 & 72 \\
\hline$\ldots$ & $\ldots$ & $\ldots$ & $\ldots$ & $\ldots$ & $\ldots$ & $\ldots$ & $\ldots$ & $\ldots$ & $\ldots$ \\
\hline
\end{tabular}
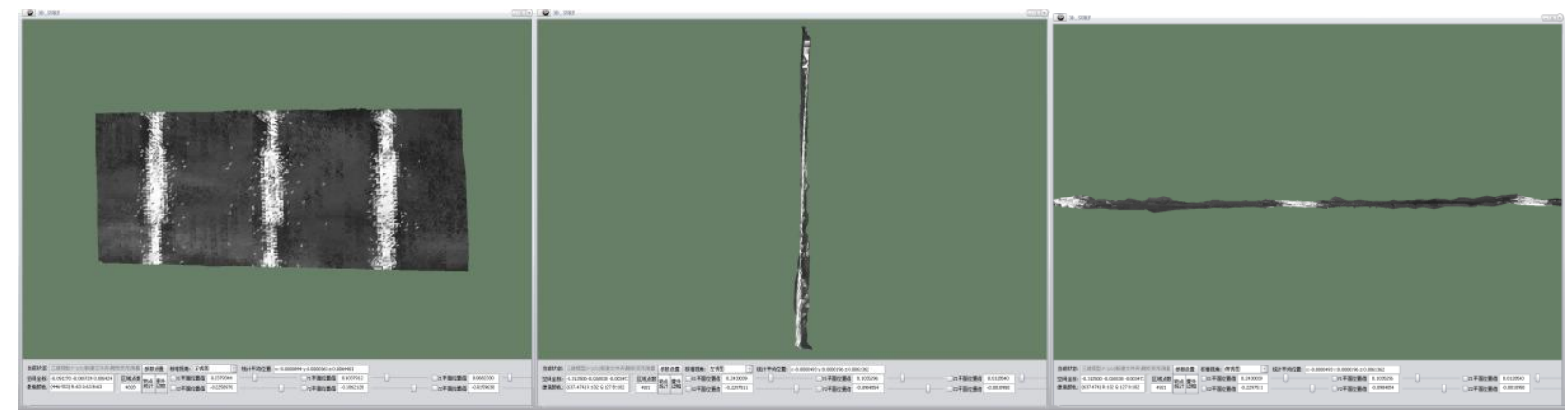

Fig. 10 Front view, left view, top view of the solar wing panel model

From Fig. 10 shown above, general morphology characteristics of the panel can be recognized. The blur in the model mainly result from the error in the image at some point. After setting the frame the quantitative result showed that, the number of points in the area is 4922, and the average position is " $\mathrm{x}:-0.000 \mathrm{y}: 0.000 \mathrm{z}: 0.006$ ". The synthesized plane is " $-0.0000894 \mathrm{x}+0.0000363 \mathrm{y}-1000 \mathrm{z}-0.0064403$ $=0$ " (unit: $\mathrm{mm}$ ). The parameters of the frame plane are shown in table 4 .

Table 4 The parameters of the frame plane

\begin{tabular}{|c|c|c|c|c|c|}
\hline $\begin{array}{c}\text { X1 plane } \\
\text { parameters }\end{array}$ & $0.243 \mathrm{~mm}$ & $\begin{array}{c}\text { Y1 plane } \\
\text { parameters }\end{array}$ & $0.103 \mathrm{~mm}$ & $\begin{array}{c}\text { Z1 plane } \\
\text { parameters }\end{array}$ & $0.013 \mathrm{~mm}$ \\
\hline $\begin{array}{c}\text { X2 plane } \\
\text { parameters }\end{array}$ & $-0.230 \mathrm{~mm}$ & $\begin{array}{c}\text { Y2 plane } \\
\text { parameters }\end{array}$ & $-0.098 \mathrm{~mm}$ & $\begin{array}{c}\text { Z2 plane } \\
\text { parameters }\end{array}$ & $-0.001 \mathrm{~mm}$ \\
\hline
\end{tabular}

The difference between $\mathrm{Z} 1$ plane and $\mathrm{Z} 2$ plane illustrates the height difference between the front and back surface of the solar wing is $0.014 \mathrm{~mm}$. Generally $0.1 \mathrm{~mm}$ are considered as small deformation for the panel, so we can think the solar wing in this experiment is flat. 


\section{Summary}

In this paper, we measured the deformation of solar wing panel using binocular camera measurement system which can calculate the geometry information of 3D object through intersection measurement. We also researched the key technology of binocular stereo vision camera measurement system including imaging principle, imaging measurement system calibration, and image feature point matching.

A feasible optimization scheme of epipolar constraint is proposed, which combines the correlation matching algorithm and the least square matching algorithm, improving the computational efficiency. In the paper, we realized each function module through programming. Experiments were carried out to reconstruct the 3D model and measure the deformation of solar wing panel. The experimental results showed that the solar wing panel deformation measurement system based on binocular stereo vision proposed in this paper is of non-contact and of high accuracy, can accurate measure solar wing panel deformation which is of great significance for further structural strength analysis, vibration suppression and motion control.

\section{Acknowledgements}

This research was supported by National Natural Science Foundation of China (Grant No. 11472302) and National Key Scientific Instrument and Equipment Development Project (Grant No. 2013YQ140517).

\section{References}

[1] YU Q F, SHANG Yang. Videometrics: principles and researches [M]. Beijing: Science Press, 2009. (in Chinese).

[2] Z. Zhang, "A flexible new technique for camera calibration", IEEE Transactions on Pattern Analysis and Machine Intelligence,22(11):1330-1334, 2000.

[3] F. Ackerman. Digital image correlation: Performance and potential application in photogrammetry. Photogrammetric Record, 11(64):429-439, 1984.

[4] YU Q F, Shang Y, Zhou J, et al. Monocular trajectory intersection method for 3D motion measurement of a point target [J]. Sci China Ser E-Tech Sci, 2009, 52(12): 3454 - 3463.

[5] B. Micusik, Trajectory reconstruction from non-overlapping surveillance cameras with relative depth ordering constraints [C]. ICCV, 2011: 922 - 928.

[6] Avidan, S. and A. Shashua. "Trajectory triangulation 3D reconstruction of moving points from a monocular image dequence." IEEE TPAMI, 22(4), 2000.

[7] Amir Amihood, Cole Richard, Hariharan Ramesh. Overlap matching [J]. Information and Computation, 2003, 181 (1):57-61. 